Please cite this paper as follows:

Jeng-Tzong Chen, Hong-Ki Hong, and Chau-Shioung Yeh, Modal Reaction Method for Modal Participation Factors in Support Motion Problems, Communications in Numerical Methods in Engineering, Vol.9, pp.479-490, 1995. 


\title{
MODAL REACTION METHOD FOR MODAL PARTICIPATION FACTORS IN SUPPORT MOTION PROBLEMS
}

\author{
J. T. CHEN \\ Department of Harbor and River Engineering, Taiwan Ocean University, Keelung, Taiwan \\ AND \\ H.-K. HONG AND C. S. YEH \\ Department of Civil Engineering, Taiwan University, Taipei, Taiwan
}

\begin{abstract}
SUMMARY
An efficient method, called the modal reaction method, for calculating the modal participation factors for support motion problems is proposed. It is found that modal reactions at supports proportionally describe the magnitudes of the modal participation factors. Thus, the modal participation factor for support motion problems can be calculated very efficiently, saving more than 99 per cent, for the given example with 640 degreees of freedom, of the CPU time compared with using the popular quasistatic decomposition method.
\end{abstract}

KEY wORDS Modal reaction method; modal participation factor, support motion; discrete system; modal equivalent mass; quasi-static decomposition

\section{INTRODUCTION}

In solving dynamic problems, either direct transient analysis or modal analysis can be utilized. For the case of modal analysis, the total response is obtained by superimposing the contributions of natural modes, and each of the generalized co-ordinates represents the weight of the contribution made by the corresponding mode. While the modal co-ordinate and the modal equivalent mass ratio for base shear play the roles of modal outputs for displacement and base shear, respectively, the modal participation factor plays the role of modal input by distributing external excitations to the corresponding mode. The derivations of these factors due to body force, boundary force and support excitations are well known in the literature. ${ }^{1}$ When the excitation is body force or boundary force, the modal displacement at the application point of the body force or the boundary force governs the magnitude of the modal participation factor. Figure 1(a) illustrates this for a single-degree-of-freedom system. Therefore, if a concentrated load is applied at the node of a certain mode shape, then the modal participation factor of the mode is zero.

The modal participation factor due to support motion excitations is usually determined from the quasistatic solution as Mindlin and Goodman ${ }^{2}$ proposed, even though the resulting calculation is very time-consuming and with little physical meaning. ${ }^{1}$ On the contrary, even though the quasistatic part is difficult to find, Eringen and Suhubi ${ }^{3}$ managed to obtain, free from the calculation of the quasistatic part, the general dynamic solution of the total response for a continuous system. Motivated by the significance of the support motion

CCC 1069-8299/95/060479-12

(C) 1995 by John Wiley \& Sons, Ltd.

Received 13 September 1993 Revised 8 September 1994 
modal porticipotion foctor of SDOF

subjected to base excltations
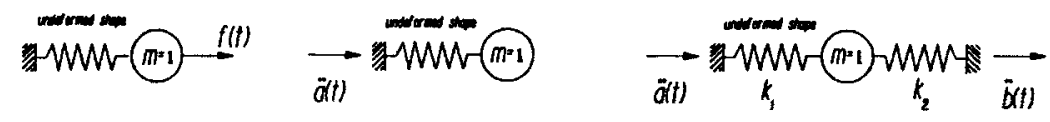

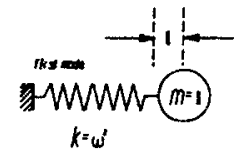

(a) force excitolion

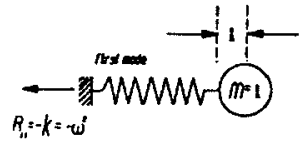

(1) single support exclition

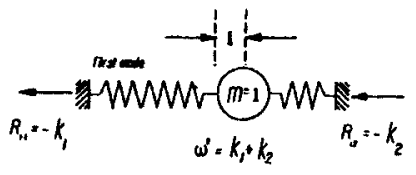

(b) multi-support excitations

Figure 1. SDOF model subjected to (a) force excitation, (b) single support excitation and (c) multiple support excitations

problems in earthquake engineering, we synthesize and extend their ideas ${ }^{3}$ to discrete multidegrees-of-freedom structural systems, and find that the modal participation factor is proportional to the modal reaction, thus revealing a clear physical meaning and facilitating calculation.

\section{MODAL FORMULATION FOR SUPPORT MOTION IN DISCRETE SYSTEMS}

Consider the discrete system with the governing equation:

$$
[\mathbf{M}]\{\dot{\mathbf{U}}\}+[\mathbf{C}][\dot{\mathbf{U}}\}+[\mathbf{K}]\{\mathbf{U}\}=\{\mathbf{P}(t)\}
$$

where $[\mathrm{M}]$ is mass, $[\mathrm{C}]$ is damping and $[\mathrm{K}]$ is stiffness, all being matrices of order $N$, the total number of degrees of freedom, $\{\mathbf{U}\}$ is displacement and $\{\mathbf{P}\}$ is force, all being column matrices. We decompose the degrees of freedom into two sets, the supported and the unsupported, denoted by the subscripts $r$ and $l$. Thus, equation (1) can be rewritten as

$$
\left[\begin{array}{ll}
\mathbf{M}_{\mathrm{ll}} & \mathbf{M}_{\mathrm{r}} \\
\mathbf{M}_{\mathrm{rl}} & \mathbf{M}_{\mathrm{rr}}
\end{array}\right]\left\{\begin{array}{l}
\dot{\mathbf{U}}_{1} \\
\ddot{\mathbf{U}}_{\mathbf{r}}
\end{array}\right\}+\left[\begin{array}{ll}
\mathbf{C}_{\mathrm{ll}} & \mathbf{C}_{\mathrm{rr}} \\
\mathbf{C}_{\mathrm{rl}} & \mathbf{C}_{\mathrm{r}}
\end{array}\right]\left\{\begin{array}{l}
\dot{\mathbf{U}}_{1} \\
\dot{\mathbf{U}}_{\mathrm{r}}
\end{array}\right\}+\left[\begin{array}{ll}
\mathbf{K}_{\mathrm{n}} & \mathbf{K}_{\mathrm{rr}} \\
\mathbf{K}_{\mathrm{rl}} & \mathbf{K}_{\mathrm{rr}}
\end{array}\right]\left\{\begin{array}{l}
\mathbf{U}_{1} \\
\mathbf{U}_{\mathbf{r}}
\end{array}\right\}=\left\{\begin{array}{l}
\mathbf{P}_{1} \\
\mathbf{P}_{\mathrm{r}}
\end{array}\right\}
$$

where $\left\{\mathbf{U}_{\mathrm{r}}\right\}$ prescribes $N_{\mathrm{r}}$ support motions, $\left\{\mathbf{P}_{\mathrm{l}}\right\}$ prescribes $N_{\mathrm{l}}$ loads, $\left\{\mathbf{P}_{\mathrm{r}}\right\}$ contains the resulting $N_{\mathrm{r}}$ reaction forces, and $\left\{\mathrm{U}_{1}\right\}$ contains the resulting $N_{1}$ displacements, where $N_{\mathrm{r}}+N_{1}=N$. Since we are focusing on multisupport motion problems in earthquake engineering, the $N_{\mathrm{r}}$ entries of $\left\{\mathbf{U}_{\mathrm{r}}\right\}$ are prescribed time histories at $N_{\mathrm{r}}$ supports and it is assumed that $\left\{\mathbf{P}_{1}\right\}=0$.

The solution can be decomposed into two parts, the quasistatic part with the superscript $\mathrm{s}$ and the inertia-dynamic part with the superscript d:

$$
\{\mathbf{U}\}=\left\{\begin{array}{l}
\mathbf{U}_{\mathrm{r}} \\
\mathbf{U}_{\mathrm{r}}
\end{array}\right\}=\left\{\mathbf{U}^{\mathrm{s}}\right\}+\left\{\mathbf{U}^{\mathrm{d}}\right\}=\left\{\begin{array}{l}
\mathbf{U}_{1}^{\mathrm{s}} \\
\mathbf{U}_{\mathrm{r}}^{\mathrm{s}}
\end{array}\right\}+\left\{\begin{array}{l}
\mathbf{U}_{1}^{\mathrm{d}} \\
\mathbf{U}_{\mathrm{r}}^{\mathrm{d}}
\end{array}\right\}
$$

where, by definition, the quasistatic solution satisfies

$$
\left[\begin{array}{ll}
\mathbf{K}_{11} & \mathbf{K}_{\mathrm{r}} \\
\mathbf{K}_{\mathrm{rl}} & \mathbf{K}_{\mathrm{r}}
\end{array}\right]\left(\begin{array}{l}
\mathbf{U}_{\mathbf{s}}^{\mathrm{s}} \\
\mathbf{U}_{1}^{\mathrm{s}}
\end{array}\right\}=\left\{\begin{array}{l}
\mathbf{P}_{1}^{s} \\
\mathbf{P}_{\mathrm{r}}^{s}
\end{array}\right\}
$$


and, for the inertia-dynamic part,

$$
\begin{aligned}
& \left\{P_{1}^{d}\right\}=\{0\} \\
& \left\{U_{r}^{d}\right\}=\{0\}
\end{aligned}
$$

The eigen equation corresponding to equation (2) is

$$
-\omega_{i}^{2}\left[\begin{array}{ll}
\mathbf{M}_{\mathrm{n}} & \mathbf{M}_{\mathrm{r}} \\
\mathbf{M}_{\mathrm{rl}} & \mathbf{M}_{\mathrm{rr}}
\end{array}\right]\left\{\begin{array}{c}
\phi_{\mathrm{i}} \\
0
\end{array}\right\}+\left[\begin{array}{ll}
\mathbf{K}_{\mathrm{n}} & \mathbf{K}_{\mathrm{lr}} \\
\mathbf{K}_{\mathrm{rl}} & \mathbf{K}_{\mathrm{rr}}
\end{array}\right]\left\{\begin{array}{c}
\phi_{i} \\
0
\end{array}\right\}=\left\{\begin{array}{c}
0 \\
\mathbf{R}_{i}
\end{array}\right\}, \quad i=1,2, \ldots, N_{\mathrm{l}}
$$

where $\left\{\phi_{i}\right\}$ denotes the $i$ th mode shape with $N_{1}$ entries and $\left\{\mathbf{R}_{i}\right\}$ is the $i$ th modal reaction forces with $N_{\mathrm{r}}$ entries. The modal matrix $\left[\Phi_{\mathrm{II}}\right]$ is the collection of all the $N_{1}$ mode shapes, and the modal damping ratio $\xi_{i}$ and the $i$ th modal (or natural) frequency $\omega_{i}$ satisfy the Rayleigh damping model with coefficients $\alpha$ and $\beta$ with

that is

$$
2 \xi_{i} \omega_{i}=2 \alpha+\beta \omega_{i}^{2}, \quad i=1,2, \ldots, N_{1}
$$

$$
[\mathrm{C}]=2 \alpha[\mathbf{M}]+\beta \mathbf{K}]
$$

Denote as $\left\{Q^{d}\right\}$ the column matrix of the modal (or generalized) co-ordinates of the inertia-dynamic part. Then, the inertia-dynamic displacements have mode superposition

$$
\left\{\mathbf{U}_{1}^{\mathrm{d}}\right\}=\left[\boldsymbol{\Phi}_{11}\right]\left\{\mathbf{Q}^{\mathrm{d}}\right\} \quad\left\{\mathbf{U}^{\mathrm{d}}\right\}=\left[\boldsymbol{\Phi}_{\mathrm{N}}\right]\left\{\mathbf{Q}^{\mathrm{d}}\right\}
$$

where we have defined the augmented matrix of order $N \times N_{1}$

$$
\left[\boldsymbol{\Phi}_{\mathrm{NI}}\right]=\left[\begin{array}{c}
\boldsymbol{\Phi}_{11} \\
0
\end{array}\right]
$$

Substituting first equation (3) and then (10) into (1) and premultiplying by $\left[\Phi_{\mathrm{N}}\right]^{\mathrm{T}}$ yield

$$
\begin{aligned}
{\left[\boldsymbol{\Phi}_{\mathrm{N}}\right]^{\mathrm{T}}[\mathbf{M}]\left[\boldsymbol{\Phi}_{\mathrm{N} 1}\right]\left[\ddot{\mathbf{Q}}^{\mathrm{d}}(t)\right\}+\left[\boldsymbol{\Phi}_{\mathrm{N} 1}\right]^{\mathrm{T}}[\mathbf{C}]\left[\boldsymbol{\Phi}_{\mathrm{N} 1}\right]\left\{\dot{\mathbf{Q}}^{\mathrm{d}}(t)\right\}+\left[\boldsymbol{\Phi}_{\mathrm{N} 1}\right]^{\mathrm{T}}[\mathbf{K}]\left[\boldsymbol{\Phi}_{\mathrm{N}}\right]\left\{\mathbf{Q}^{\mathrm{d}}(t)\right\} } \\
=-\left[\boldsymbol{\Phi}_{\mathrm{N} 1}\right]^{\mathrm{T}}[\mathbf{M}]\left\{\ddot{U}^{\mathrm{s}}\right\}-\left[\boldsymbol{\Phi}_{\mathrm{N}}\right]^{\mathrm{T}}[\mathbf{C}]\left(\dot{U}^{s}\right\}
\end{aligned}
$$

Note that, in the present formulation, there is no difficulty in retaining the $\left[\Phi_{\mathrm{N}}\right]^{\mathrm{T}}[\mathbf{C}]\left\{\dot{U}^{\mathrm{s}}\right\}$ term in equation (12), in contrast to the paper in which the term was always neglected. ${ }^{4}$ The modal co-ordinate, $Q_{i}^{\mathrm{d}}$, satisfies

$$
\ddot{Q}_{i}^{\mathrm{d}}+2 \xi_{i} \omega_{i} \dot{Q}_{i}^{\mathrm{d}}+\omega_{i}^{\mathrm{z}} Q_{i}^{\mathrm{d}}=-\left\{\begin{array}{c}
\phi_{i} \\
0
\end{array}\right\}^{\mathrm{T}}[\mathbf{M}]\left\{\ddot{U}^{\mathrm{s}}\right\}-\left\{\begin{array}{c}
\phi_{i} \\
0
\end{array}\right\}^{\mathrm{T}}[\mathbf{C}]\left[\dot{U}^{\mathrm{s}}\right\}
$$

for the modal co-ordinate of the inertia-dynamic part $Q_{i}^{d}$.

\section{APPLICATION OF BETTI'S LAW FOR DISCRETE SYSTEM}

Since $\left\{\mathbf{P}_{1}\right\}=\left\{\mathbf{P}_{1}^{s}\right\}=\{0\}$, we obtain, from equation (4),

$$
\left\{\mathbf{U}_{1}^{s}\right\}=-\left[\mathbf{K}_{11}\right]^{-1}\left[\mathbf{K}_{\mathrm{lr}}\right\}\left\{\mathbf{U}_{\mathrm{r}}^{\mathrm{s}}\right\}
$$

We now apply Betti's law for discrete systems ${ }^{5}$ to prove

$$
-\omega_{i}^{2}\left\{\phi_{i}\right\}^{\mathrm{T}}\left[\mathbf{M}_{11} \mathbf{M}_{\mathrm{Ir}}\right]\left\{\mathbf{U}^{\mathrm{s}}\right\}=-\omega_{i}^{2}\left\{\begin{array}{c}
\phi_{i} \\
0
\end{array}\right\}^{\mathrm{T}}[\mathbf{M}]\left[\mathbf{U}^{\mathrm{s}}\right\}=\left\{\mathbf{R}_{i}\right\}^{\mathrm{T}}\left\{\mathbf{U}_{\mathrm{r}}^{s}\right\}
$$

Based on the eigen-equation (7), we obtain the $i$ th modal reaction as follows:

$$
\left\{\mathbf{R}_{i}\right\}=-\omega_{i}^{2}\left[\mathbf{M}_{\mathrm{r}}\right]\left\{\phi_{i}\right\}+\left[\mathbf{K}_{\mathrm{r}}\right]\left\{\phi_{i}\right\}
$$


Then, inserting equation (16) into the right-hand side of equation (15), we obtain

$$
\begin{aligned}
\left\{\mathbf{R}_{i}\right\}^{\mathrm{T}}\left\{\mathbf{U}_{\mathrm{r}}^{s}\right\} & =\left\{-\omega_{i}^{2}\left[\mathbf{M}_{\mathrm{r}}\right]\left\{\phi_{i}\right\}+\left[\mathbf{K}_{\mathrm{rl}}\right]\left\{\phi_{i}\right\}\right\}^{\mathrm{T}}\left\{\mathbf{U}_{\mathrm{r}}^{s}\right\} \\
& =\left\{-\omega_{i}^{2}\left[\phi_{i}\right\}^{\mathrm{T}}\left[\mathbf{M}_{\mathrm{rr}}\right]+\left\{\phi_{i}\right\}^{\mathrm{T}}\left[\mathbf{K}_{\mathrm{lr}}\right]\right\}\left\{\mathbf{U}_{\mathrm{r}}^{\mathrm{s}}\right\}
\end{aligned}
$$

Substitution of equation (14) into the left-hand side of equation (15) yields

$$
-\omega_{i}^{2}\left\{\phi_{i}\right\}^{\mathrm{T}}\left[\mathbf{M}_{11} \mathbf{M}_{\mathrm{lr}}\right]\left\{\mathbf{U}^{\mathrm{s}}\right\}=\omega_{i}^{2}\left\{\left\{\phi_{i}\right\}^{\mathrm{T}}\left[\mathbf{M}_{\mathrm{l}}\right]\left[\mathbf{K}_{\mathrm{ll}}\right]^{-1}\left[\mathbf{K}_{\mathrm{lr}}\right]-\left\{\phi_{i}\right\}^{\mathrm{T}}\left[\mathbf{M}_{\mathrm{lr}}\right]\right\}\left\{\mathbf{U}_{\mathrm{r}}^{s}\right\}
$$

After comparing equation (17) with (18), to derive (15) is equivalent to finding

$$
\omega_{i}^{2}\left\{\phi_{i}\right\}^{\mathrm{T}}\left[\mathbf{M}_{11}\right]\left[\mathbf{K}_{\mathrm{ll}}\right]^{-1}\left[\mathbf{K}_{\mathrm{lr}}\right]\left\{\mathbf{U}_{\mathrm{r}}^{\mathrm{s}}\right\}=\left\{\phi_{i}\right\}^{\mathrm{T}}\left[\mathbf{K}_{\mathrm{lr}}\right]\left\{\mathbf{U}_{\mathrm{r}}^{\mathrm{s}}\right\}
$$

From the eigensystem of equation (7), we have

$$
-\omega_{i}^{2}\left[\mathbf{M}_{11}\right]\left\{\phi_{i}\right\}+\left[\mathbf{K}_{11}\right]\left\{\phi_{i}\right\}=\{0\}
$$

Taking the transpose of equation (20) and postmultiplying with $\left[K_{11}\right]^{-1}\left[K_{1 r}\right]\left\{\mathbf{U}_{\mathrm{r}}^{s}\right\}$, we have

$$
-\omega_{i}^{2}\left\{\phi_{i}\right\}^{\mathrm{T}}\left[\mathbf{M}_{11}\right]\left[\mathbf{K}_{\mathrm{n}}\right]^{-1}\left[\mathbf{K}_{\mathrm{lr}}\right]\left(\mathbf{U}_{\mathrm{r}}^{\mathrm{s}}\right\}+\left\{\phi_{i}\right\}^{\mathrm{T}}\left[\mathbf{K}_{\mathrm{ll}}\right]\left[\mathbf{K}_{\mathrm{ll}}\right]^{-1}\left[\mathbf{K}_{\mathrm{lr}}\right]\left\{\mathbf{U}_{\mathrm{r}}^{\mathrm{s}}\right\}=0
$$

Therefore, we have derived equation (19) and hence equation (15).

\section{TRANSIENT RESPONSE USING MODAL REACTION METHOD}

Now considering equations (3), (6) and (13), we can express the quasistatic part as

$$
\begin{aligned}
\left\{\mathbf{U}^{s}\right\} & =\left[\begin{array}{c}
-\mathbf{K}_{11}^{-1} \mathbf{K}_{\mathrm{lr}} \\
\mathbf{I}
\end{array}\right]\left\{\mathbf{U}_{\mathrm{r}}\right\} \\
& \equiv\left[\begin{array}{llllll}
\left\{\mathbf{G}_{1}\right\} & \left\{\mathbf{G}_{2}\right\} & \cdots & \left\{\mathbf{G}_{j}\right\} & \cdots & \left\{\mathbf{G}_{N_{\mathrm{r}}}\right\}
\end{array}\right]\left\{\begin{array}{c}
U_{\mathrm{r} 1}(t) \\
U_{\mathrm{r} 2}(t) \\
\vdots \\
U_{\mathrm{r} j}(t) \\
\vdots \\
U_{\mathrm{rNr}}(t)
\end{array}\right)
\end{aligned}
$$

where $U_{\mathrm{r} j}(t)$ is the $j$ th support history of the $r$ set and $\left\{\mathbf{G}_{j}\right\}$ denotes the quasistatic influence vector for $\left\{U^{s}\right\}$ when only the $j$ th entry of $\left\{U_{s}\right\}$ is 1 ; otherwise it is zero. Equation (22) can be rewritten as

$$
\left\{\mathbf{U}^{\mathrm{s}}\right\}=\sum_{j=1}^{N_{\mathrm{r}}}\left\{\mathbf{G}_{j}\right\} U_{\mathrm{r} j}(t)
$$

Substituting equation (23) into (13), we have

$$
\begin{aligned}
\ddot{Q}_{i}^{\mathrm{d}}+2 \xi_{i} \omega_{i} \dot{Q}_{i}^{\mathrm{d}}+\omega_{i}^{2} Q_{i}^{\mathrm{d}} & =\sum_{j=1}^{N_{\mathrm{r}}}\left(-\left\{\begin{array}{c}
\phi_{i} \\
0
\end{array}\right\}^{\mathrm{T}}[\mathbf{M}]\left\{\mathbf{G}_{j}\right\} \ddot{\mathbf{U}}_{\mathrm{r} j}(t)-\left\{\begin{array}{c}
\phi_{i} \\
0
\end{array}\right\}^{\mathrm{T}}[\mathbf{C}]\left\{\mathbf{G}_{j}\right\} \dot{\mathbf{U}}_{\mathrm{r} j}(t)\right) \\
& =-\sum_{j=1}^{N_{\mathrm{r}}}\left(\Gamma_{i j}^{\mathrm{d}} \ddot{U}_{\mathrm{r} j}(t)\right)-2 \alpha \sum_{j=1}^{N_{\mathrm{t}}}\left(\Gamma_{i j}^{\mathrm{d}} \dot{U}_{\mathrm{r} j}(t)\right)
\end{aligned}
$$


after using equations (4) and (9), where $\Gamma_{i j}^{d}$ is the $i$ th modal participation factor for the inertia-dynamic part $Q_{i}^{\mathrm{d}}$ subjected to the $j$ th support motion $\ddot{U}_{\mathrm{rj}}(t)$, defined as

$$
\Gamma_{i j}^{\mathrm{d}} \equiv\left\{\begin{array}{c}
\phi_{i} \\
0
\end{array}\right\}^{\mathrm{T}}[\mathbf{M}]\left\{\mathbf{G}_{j}\right\}, \quad i=1,2, \ldots, N_{\mathrm{l}}, \quad j=1,2, \ldots, N_{\mathrm{r}}
$$

By substituting the particular selection,

$$
\begin{gathered}
\left\{\mathbf{U}^{s}\right\}=\left\{\mathbf{G}_{j}\right\} \\
\left\{\mathbf{U}_{\mathrm{r}}\right\}=\left\{\mathbf{U}_{\mathrm{r}}^{s}\right\}=\left\{\begin{array}{c}
0 \\
0 \\
\vdots \\
U_{\mathrm{r} j}(t) \\
\vdots \\
0
\end{array}\right)=\left(\begin{array}{c}
0 \\
0 \\
\vdots \\
1 \\
\vdots \\
0
\end{array}\right)
\end{gathered}
$$

into equation (15), we obtain the general equality

$$
-\omega_{i}^{2}\left\{\begin{array}{c}
\phi_{i} \\
0
\end{array}\right\}^{\mathrm{T}}[\mathbf{M}]\left\{\mathbf{G}_{j}\right\}=R_{i j}, \quad i=1,2, \ldots, N_{1}, \quad j=1,2, \ldots, N_{\mathrm{r}}
$$

where $R_{i j}$ is the $j$ th entry of the $i$ th modal reaction, i.e., the $j$ th support reaction of the $i$ th mode. From equations (25) and (28), we have

$$
\Gamma_{i j}^{\mathrm{d}}=\frac{R_{i j}}{\left(-\omega_{i}^{2}\right)}, \quad i=1,2, \ldots, N_{1}, \quad j=1,2, \ldots, N_{\mathrm{r}}
$$

which expresses that the modal participation factor for the inertia-dynamic part is the modal reaction divided by the negative of the modal frequency squared.

Nevertheless, on the other hand, the quasistatic part is still present in the total response of equation (3), and it seems that the calculation of the quasistatic solution could not be omitted in deriving the total response, although we can determine the modal participation factor without the quasistatic solution by using equation (29). However, if we define

$$
\left\{\mathbf{Q}^{\mathrm{s}}\right\} \equiv\left[\boldsymbol{\Phi}_{\mathrm{Nl}}\right]^{\mathrm{T}}[\mathbf{M}]\left\{\mathbf{U}^{\mathrm{s}}\right\}=-\operatorname{diag}\left(1 / \omega_{1}^{2}, 1 / \omega_{2}^{2}, \ldots, 1 / \omega_{N_{1}}^{2}\right)\left\{R_{1} \ldots R_{i} \ldots R_{1}\right\}^{\mathrm{T}}\left\{\mathbf{U}_{\mathrm{r}}\right\}
$$

owing to equation (9), the quasistatic solution can be expanded in terms of natural modes as follows:

$$
\left\{\mathbf{U}^{\mathrm{s}}\right\}=\left[\Phi_{M}\right]\left\{\mathbf{Q}^{\mathrm{s}}\right\}=-\left[\Phi_{N_{1}}\right] \operatorname{diag}\left(1 / \omega_{1}^{2}, 1 / \omega_{2}^{2}, \ldots, 1 / \omega_{N_{1}}^{2}\right)\left\{R_{1} \ldots R_{i} \ldots R_{1}\right\}^{\mathrm{T}}\left\{\mathbf{U}_{\mathrm{r}}\right\}
$$

After merging the quasistatic part into the modal (or generalized) co-ordinates of the quasistatic part $\left\{Q^{s}\right\}$ by equation (30), the solution can be written in terms of a more generalized one, the modal co-ordinates $\{Q\}$ as follows:

$$
\left\{\mathbf{U}_{1}\right\}=\left\{\mathbf{U}_{1}^{s}\right\}+\left[\boldsymbol{\Phi}_{11}\right]\left\{\mathbf{Q}^{\mathrm{d}}\right\}=\left[\boldsymbol{\Phi}_{11}\right]\left\{\mathbf{Q}^{\mathrm{s}}\right\}+\left[\boldsymbol{\Phi}_{11}\right]\left(\mathbf{Q}^{\mathrm{d}}\right\}=\left[\boldsymbol{\Phi}_{11}\right]\{\mathbf{Q}\}
$$

where $\{\mathbf{Q}\}=\left\{\mathbf{Q}^{s}\right\}+\left\{\mathbf{Q}^{\mathrm{d}}\right\}$. Substituting equations (32) and (9) into (13), for the $i$ th modal coordinate $Q_{i}\left(i=1,2, \ldots, N_{1}\right)$, we have

$$
\begin{aligned}
\ddot{Q}_{i}+2 \xi_{i} \omega_{i} \dot{Q}_{i}+\omega_{i}^{2} Q_{i} & =\sum_{j=1}^{N_{\mathrm{r}}} R_{i j}\left(-\beta \dot{U}_{\mathrm{r} j}(t)-U_{\mathrm{r} j}(t)\right) \\
& =-\beta \sum_{j=1}^{N_{\mathrm{r}}}\left(\Gamma_{i j} \dot{U}_{\mathrm{r} j}(t)\right)-\sum_{j=1}^{N_{\mathrm{r}}}\left(\Gamma_{i j} U_{\mathrm{rj}}(t)\right)
\end{aligned}
$$


where the $i$ th modal participation factor $\Gamma_{i j}$ subjected to $j$ th support motion $U_{\mathrm{r} j}(t)$ is defined as

$$
\Gamma_{i j} \equiv R_{i j}, \quad i=1,2, \ldots, N_{1}, \quad j=1,2, \ldots, N_{r}
$$

which simply expresses that the modal participation factor is just the modal reaction.

For the types of the time history records of support motions $\ddot{U}_{\mathrm{rj}}(t), \dot{U}_{\mathrm{rj}}(t)$ and $U_{\mathrm{rj}}(t)$, the corresponding modal participation factors $\Gamma_{i j}^{\mathrm{d}}$ and $\Gamma_{i j}$ with respect to $Q_{i}^{\mathrm{d}}$ and $Q_{i}$, respectively, are shown in Table I.

Using the Duhamel integral for equations (24) and (33), we have

$$
\begin{aligned}
& Q_{i}^{\mathrm{d}}(t)=\frac{1}{\omega_{i} \sqrt{1-\xi_{i}^{2}}} \int_{0}^{t} \mathrm{e}^{-\xi_{i} \omega_{i}(t-\tau)} \sin \left(\omega_{\mathrm{i}}^{\mathrm{d}}(t-\tau)\right) \sum_{j=1}^{N_{\mathrm{t}}}\left(\frac{R_{i j}}{\omega_{i}^{2}}\left(\ddot{U}_{\mathrm{rj}}(\tau)+2 \alpha \dot{U}_{\mathrm{rj}}(\tau)\right)\right) \mathrm{d} \tau \\
& Q_{i}(t)=\frac{-1}{\omega_{i} \sqrt{1-\xi_{i}^{2}}} \int_{0}^{t} \mathrm{e}^{-\xi_{\mathrm{i}} \omega_{\mathrm{l}}(t-\tau)} \sin \left(\omega_{\mathrm{i}}^{\mathrm{d}}(t-\tau)\right) \sum_{j=1}^{N_{\mathrm{t}}}\left(R_{i j}\left(\beta \dot{U}_{\mathrm{rj}}(\tau)+U_{\mathrm{rj}}(\tau)\right)\right) \mathrm{d} \tau
\end{aligned}
$$

respectively. The displacement response can be derived according to equation (32) by applying equations (35) and (36).

In earthquake resistant design, the partial sum of the series of modal equivalent mass ratios for base shear plays the role of determining the modes needed, since

$$
\sum_{j=1}^{N_{\mathrm{r}}} \sum_{i=1}^{N_{1}} m_{i j}=M_{q}
$$

where $M_{q}$ is normalized quasi-static mass ${ }^{8}$ and the $i$ th modal equivalent mass ratio subjected to the $j$ th support motion can be calculated by the modal reaction as follows:

$$
m_{i j}=\left(\Gamma_{i j}^{d}\right)^{2}=\frac{\left(R_{i j}\right)^{2}}{\omega_{i}^{4}}, \quad i=1,2, \ldots, N_{\mathrm{l}}, \quad j=1,2, \ldots, N_{\mathrm{r}}
$$

\begin{tabular}{|c|c|c|c|c|}
\hline \multicolumn{2}{|c|}{$\begin{array}{l}\text { Modal participation } \\
\text { factor } \Gamma_{i j}\end{array}$} & $\ddot{U}_{\mathrm{Ij}}(t)$ & $\dot{U}_{\mathrm{rj}}(T)$ & $U_{\mathrm{rj}}(t)$ \\
\hline$Q_{i}^{\mathrm{d}}$ & domain & $\left\{\begin{array}{c}\phi_{i} \\
0\end{array}\right\}^{\mathrm{T}}[\mathbf{M}]\left\{\mathbf{G}_{j}\right\}$ & $2 a\left\{\begin{array}{c}\phi_{i} \\
0\end{array}\right\}^{\mathrm{T}}[\mathbf{M}]\left\{\mathbf{G}_{j}\right\}$ & 0 \\
\hline \multirow{3}{*}{$Q_{i}$} & boundary & $\frac{R_{i j}}{\left(-\omega_{i}^{2}\right)}$ & $2 a \frac{R_{i j}}{\left(-\omega_{i}^{2}\right)}$ & 0 \\
\hline & domain & 0 & $-\beta \omega_{i}^{2}\left\{\begin{array}{c}\phi_{i} \\
0\end{array}\right\}^{\mathrm{T}}[\mathbf{M}]\left\{\mathbf{G}_{j}\right\}$ & $-\omega_{i}^{2}\left\{\begin{array}{l}\phi_{i} \\
0\end{array}\right\}^{\mathrm{T}}[\mathbf{M}]\left\{\mathbf{G}_{j}\right\}$ \\
\hline & boundary & 0 & $\beta R_{i j}$ & $R_{i j}$ \\
\hline
\end{tabular}

Table I. Comparison of modal participation factor by quasistatic decomposition and modal reaction methods 


\section{NUMERICAL EXAMPLES}

Example 1. A single-degree-of-freedom system subjected to single and multiple support excitations

As shown in Figure 1(b), the new point of view for the modal participation factor is checked by a single-degree-of-freedom system. By the definition of equation (29), the modal participation factor is:

single support excitation:

$$
\Gamma_{11}^{\mathrm{d}}=\frac{R_{11}}{\left(-\omega_{1}^{2}\right)}=\frac{\omega_{1}^{2}}{\omega_{1}^{2}}=1
$$

multiple support excitations:

$$
\begin{aligned}
& \Gamma_{11}^{\mathrm{d}}=\frac{R_{11}}{\left(-\omega_{1}^{2}\right)}=\frac{k_{1}}{k_{1}+k_{2}} \\
& \Gamma_{12}^{\mathrm{d}}=\frac{R_{12}}{\left(-\omega_{1}^{2}\right)}=\frac{k_{2}}{k_{1}+k_{2}}
\end{aligned}
$$

which just match the modal participation factors in structural dynamics. ${ }^{6}$

\section{Example 2. Cantilever beam}

Consider a multi-degrees-of-freedom system with 20 beam elements subjected to base excitation. The modal participation factor determined by the quasistatic decomposition method and the modal reaction method are summarized in Table II. It is found that using the modal reaction method CPU time consumed in VAX 8600 is only one-sixth of that using the quasistatic decomposition method as shown in Table II. The partial sum of the series of the modal equivalent mass ratios for base shear against the mode number is shown in Figure 2.

\section{Example 3. Flexural beam}

Extending the single input to multiple support excitations, the long bridge model is discretized into 20 beam elements. The modal participation factors of the quasistatic decomposition method

Table II. Comparison for cantilever beam subjected to base excitation

\begin{tabular}{cccc}
\hline & & \multicolumn{2}{c}{ Discrete system (20 elements) } \\
\cline { 3 - 4 } Continuous system & Quasistatic decomposition & Modal reaction method \\
\hline & Exact solution & $\left\{\phi_{i i}\right\}^{\mathrm{T}}[\mathbf{M}]\left[\mathbf{G}_{j}\right\}$ & $\frac{R_{i 1}}{\left(-\omega_{\mathbf{j}}^{2}\right)}$ \\
& & & 1.568 \\
$\Gamma_{11}$ & 1.566 & 1.569 & -0.868 \\
$\Gamma_{21}$ & -0.867 & -0.868 & 0.509 \\
$\Gamma_{31}$ & 0.509 & 0.509 & -0.364 \\
$\Gamma_{41}$ & -0.364 & -0.364 & 0.01 \\
\hline
\end{tabular}




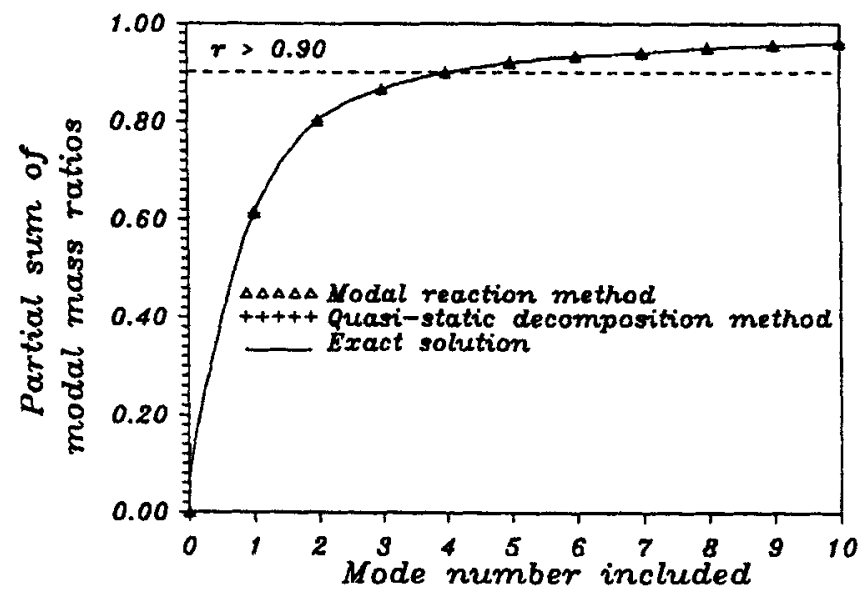

Figure 2. Partial sum of modal mass ratios against mode number included

and the modal reaction method agree satisfactorily, as shown in Table III. The increasing rate of the CPU time for the quasistatic decomposition method is higher than the modal reaction method as shown in Table III. It is shown that only 1 per cent CPU time is needed for the example with 640 degrees of freedom using the present method in comparison with the quasistatic decomposition method. In Figure 3, the transient responses at the middle point to the in-phase motions $a(t)=b(t)=0.01 \mathrm{e}^{-0.1 t} \sin (1.2 \pi t)$ at both ends using the quasistatic decomposition method and the modal reaction method are shown and compared with other solutions. ${ }^{7,9}$ The results are very satisfactory.

\section{Example 4. Frame with a rigid slab}

The last example shows the frame structure with a rigid slab subjected to in-phase and out-of-phase support motions as shown in Figure 4. The modal participation factors are shown in Table IV and are

Table III. Comparison for flexural beam subjected to multisupport motions

\begin{tabular}{lccc}
\hline & & \multicolumn{2}{c}{ Discrete system (20 elements) } \\
\cline { 3 - 4 } & Continuous system & Quasistatic decomposition & Modal reaction method \\
\hline & & $\left\{\phi_{i}\right\}^{\mathrm{T}}[\mathbf{M}]\left\{\mathbf{G}_{j}\right\}$ & $\frac{R_{i 1}}{\left(-\omega_{\mathrm{i}}^{2}\right)}$ \\
& Exact solution & & 170.82 \\
$\Gamma_{11}$ & 170.82 & 170.82 & 85.41 \\
$\Gamma_{21}$ & 85.41 & 85.41 & -56.95 \\
$\Gamma_{31}$ & -56.94 & -56.95 & -42.71 \\
$\Gamma_{41}$ & -42.71 & -42.71 & 0.01 \\
CPU & $N=40$ & 0.06 & 0.04 \\
time, & $N=80$ & 0.42 & 0.16 \\
s & $N=160$ & 3.62 & 2.56 \\
\hline
\end{tabular}

$N=$ number of degrees of freedom 


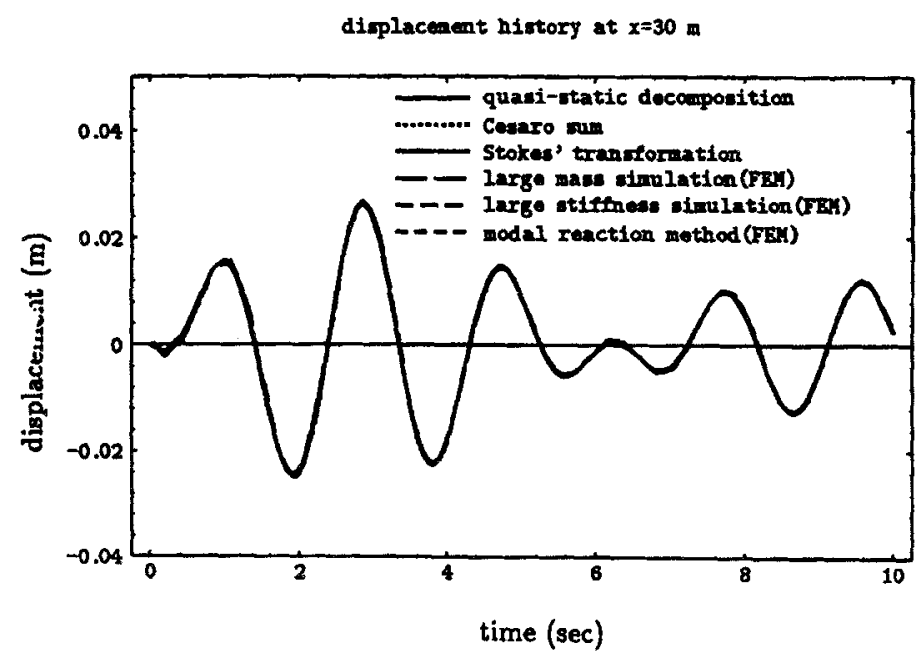

Figure 3. Displacement response at middle point for beam of Example 3
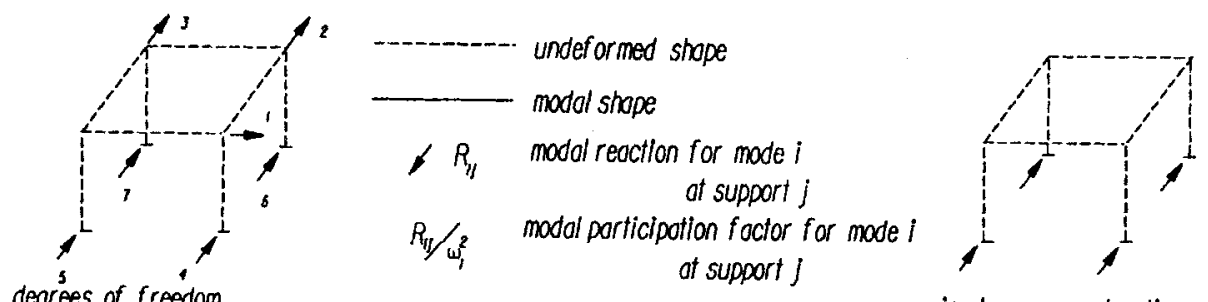

degrees of freedom

$w_{1}^{2}=\infty$
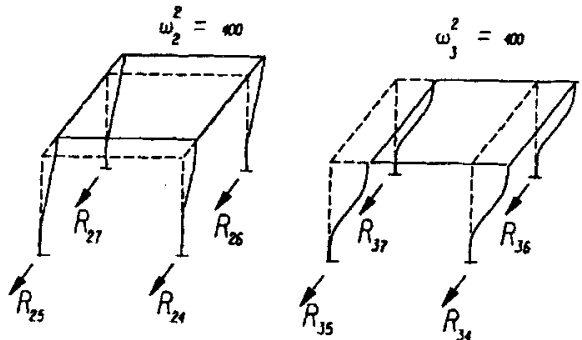

in-phose support mallons

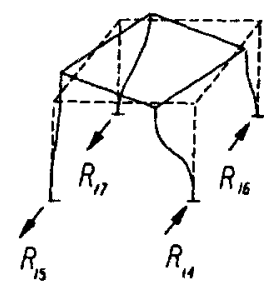

torsion mode

translational mode

translational mode out-of-phose support motions

Figure 4. Frame with a rigid slab subjected to in-phase and out-of-phase support motions 
Table IV. Modal participation factors calculated for frame with rigid slab subjected to multisupport motions

\begin{tabular}{|c|c|c|c|c|c|}
\hline \multirow{2}{*}{\multicolumn{2}{|c|}{$\begin{array}{l}\text { Modal participation } \\
\text { factor, } \Gamma_{i}^{d}\end{array}$}} & \multicolumn{3}{|c|}{$j$ th support point } & \multirow[b]{2}{*}{$j=4$} \\
\hline & & \multirow{2}{*}{$\begin{array}{r}j=1 \\
-0.10 \\
0.25 \\
0.00\end{array}$} & \multirow{2}{*}{$\begin{array}{l}j=2 \\
0.10 \\
0.25 \\
0.00\end{array}$} & \multirow{2}{*}{$\begin{array}{r}j=3 \\
-0.10 \\
0.25 \\
0.00\end{array}$} & \\
\hline $\begin{array}{l}i \text { th } \\
\text { mode }\end{array}$ & $\begin{array}{l}i=1 \\
i=2 \\
i=3\end{array}$ & & & & $\begin{array}{l}0.10 \\
0.25 \\
0.00\end{array}$ \\
\hline
\end{tabular}
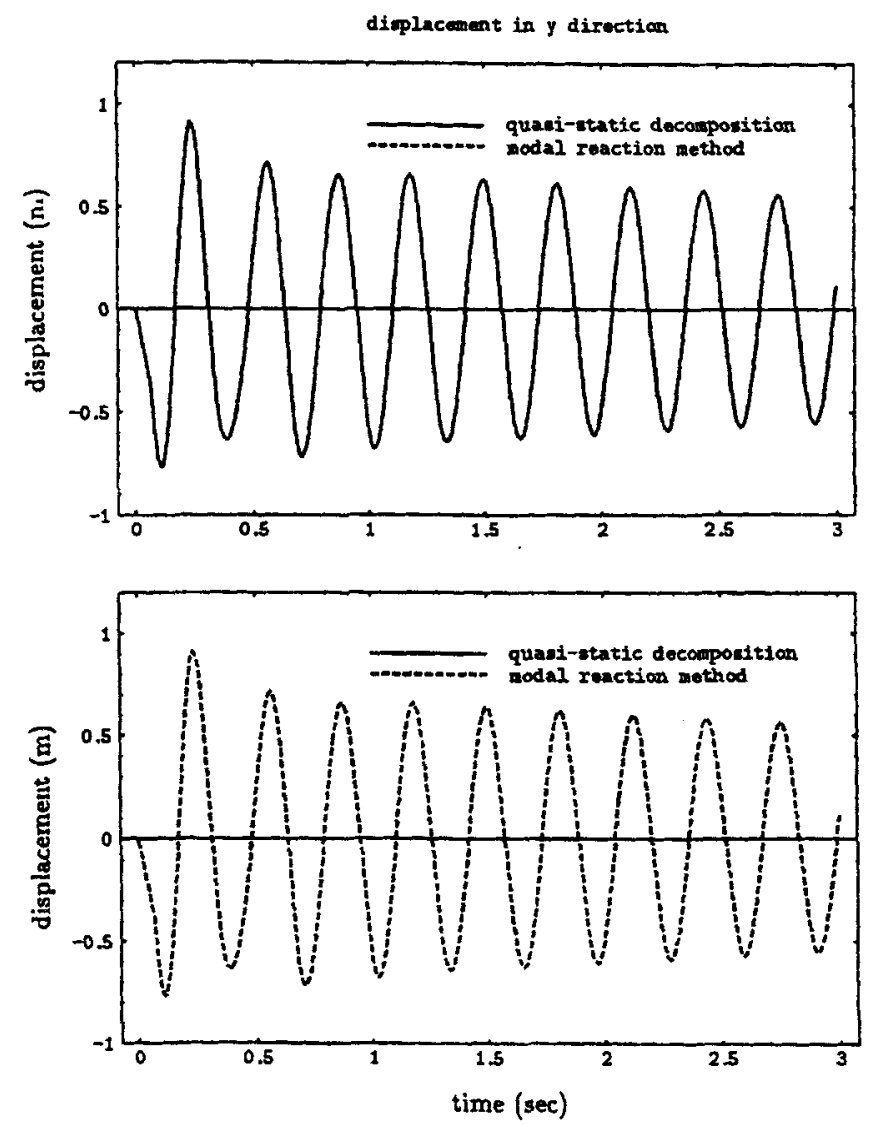

Figure 5. Displacement response of rigid slab for frame of Example 4 subjected to in-phase motions 

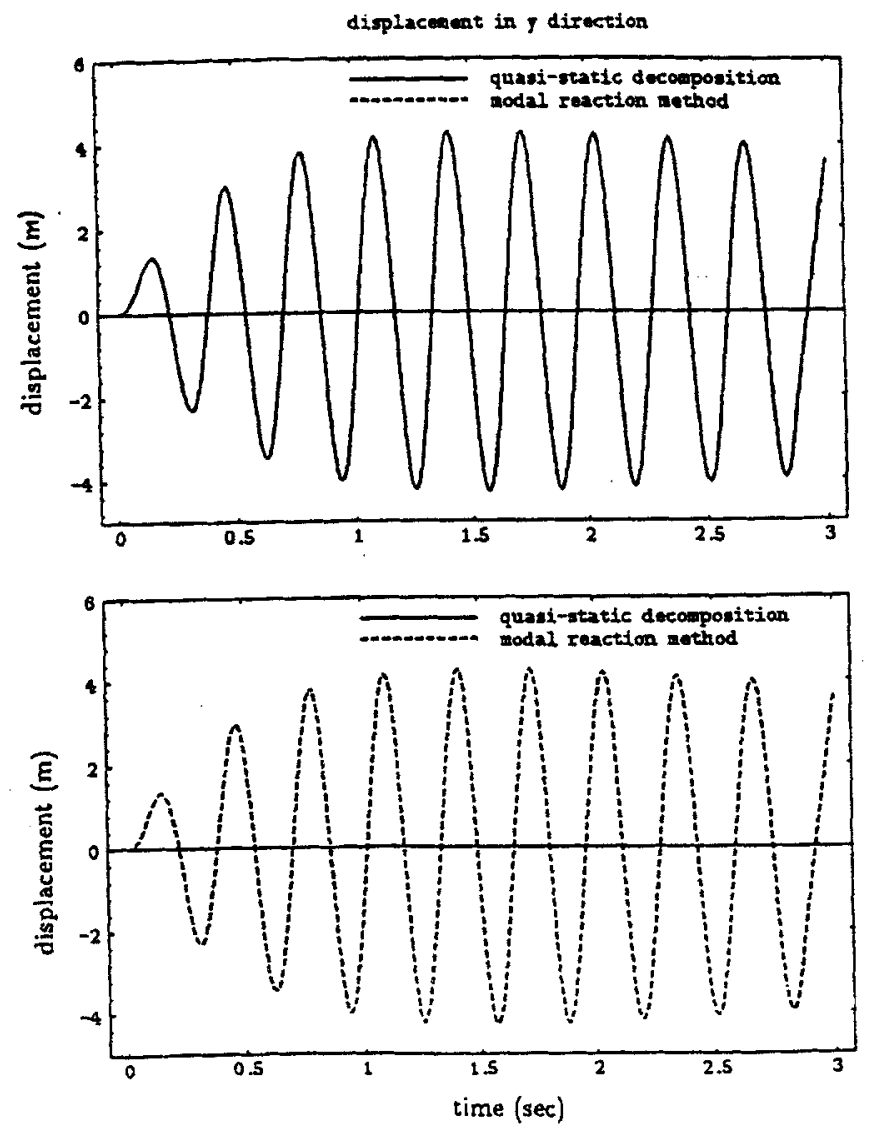

Figure 6. Displacement response of rigid slab for frame of Example 4 subjected to out-of-phase motions

the same for both methods. The transient responses of the rigid slab subjected to in-phase motions $a(t)=b(t)=0.01 \mathrm{e}^{-0.1 t} \sin (1.2 \pi t)$ and out-of-phase motions $a(t)=-b(t)=0.01 \mathrm{e}^{-0.1 t} \sin (1.2 \pi t)$ using the quasistatic decomposition method and the modal reaction method are shown in Figures 5 and 6. Good agreement is achieved for the two methods.

\section{CONCLUSIONS}

The modal reactions have been found to be proportional to the modal participation factors for support motions. Without including the computational time of the modal basis, it is shown that only 1 per cent is needed to calculate the modal participation factor when the number of degree of freedoms exceeds 640 .

\section{REFERENCES}

1. R. W. Clough and J. Penzien, Dynamics of Structures, 2nd edition, McGraw-Hill, New York, 1993.

2. R. D. Mindlin and L. E. Goodman, 'Beam vibrations with time-dependent boundary conditions', $J$. Appl. Mech., ASME, 17, 377-380 (1950).

3. A. C. Eringen and E. S. Suhubi, Elastodynamics - Linear Theory, Vol.II, Academic Press, New York, 1975. 
4. A. Der Kiureghian and A. Neuenhofer, 'Response spectrum method for multi-support seismic excitations, Earthquake eng. struct. dyn., 21, 713-740 (1992).

5. J. T. Chen and H.-K. Hong, Boundary Element Method, 2nd edition, New World Press, Taipei, Taiwan, 1992 (in Chinese).

6. G. V. Berg, Elements of Structural Dynamics, Prentice Hall, New Jersey, 1988.

7. H.-K. Hong and J. T. Chen, 'On the dual integral representation and its application to vibration problems', in C. A. Brebbia and J. J. Rencis (Eds.), Boundary Elements XV, Vol. 2, Stress Analysis, 1993, pp. 377-390.

8. J. T. Chen, 'A new point of view for quasistatic mass', in preparation, 1995.

9. J. T. Chen, H.-K. Hong, C. S. Yeh and S. W. Chyuan, 'Integral representations and regularizations for divergent series solution of a beam subjected to support motions', Earthquake Engineering and Structural Dynamics, in revised version, 1995. 